\title{
Crescimento de Diamante Dopado com Boro para Eletrodos de uso
}

\section{em Eletroquímica}

\author{
Leide Lili Gonçalves da Silva ${ }^{\mathrm{a}, \mathrm{b}^{*}}$, Evaldo José Corat ${ }^{\mathrm{a}, \mathrm{c}}$, \\ Rita de Cássia Mendes de Barros ${ }^{\mathrm{a}, \mathrm{d}}$, Vladimir Jesus Trava-Airoldi ${ }^{\mathrm{a}, \mathrm{c}}$, \\ Nélia Ferreira Leite ${ }^{\mathrm{a}}$, Koshun Iha ${ }^{\mathrm{b}}$ \\ ${ }^{a}$ Instituto Nacional de Pesquisas Espaciais, \\ Av. dos Astronautas 1758, 12227-010 São José dos Campos - SP, Brasil \\ ${ }^{\mathrm{b}}$ Centro Tecnológico de Aeronáutica, Instituto Tecnológico da Aeronáutica, \\ Departamento de Química, 12227-010 São José dos Campos - SP, Brasil \\ ${ }^{c}$ Universidade de São Francisco, Faculdade de Engenharia - Itatiba \\ ${ }^{\mathrm{d}}$ Universidade de São Paulo, Instituto de Química \\ *e-mail:leide@las.inpe.br
}

Received: February 27, 1998; Revised: April 9, 1999

\begin{abstract}
Boron-doped polycrystalline diamond films have been deposited over silicon substrate by hot-filament chemical-vapor-deposition process. A gas mixture of $0,5 \mathrm{vol} . \%$ methane and 1 vol. $\%$ methanol on hydrogen at a pressure of 50 Torr, have been used. Boric oxide dissolved in methanol have been used as the boron doping source during the diamond growth process.

Raman spectroscopy and Scanning Electron Microscopy (SEM) have been performed on the samples. A change of Raman spectra with film doping was observed. The diamond characteristic line at $1333 \mathrm{~cm}^{-1}$ down shifted and its intensity decreased as the film resistivity decreased. On the other hand, a broad peak around $1220 \mathrm{~cm}^{-1}$ appeared and its intensity increased with decreasing film resistivity.

No modifications on films morphology have been observed with different boron doping level. The grains were well-faceted with $2 \mu \mathrm{m}$ average size
\end{abstract}

Keywords: diamond, boron-doped, chemical vapour deposition, Raman spectroscopy, scanning electron microscopy

\section{Introdução}

A tecnologia de crescimento de diamante através do método CVD vem evoluindo consideravelmente nos últimos anos devido às suas excepcionais propriedades como alta condutividade térmica, largo "band gap", altas mobilidades de elétrons e lacunas, alta tensão de ruptura elétrica, dureza, transparência ótica, resistência à corrosão e inércia química $^{1-3}$.

Tradicionalmente o diamante é aplicado em dissipadores de calor, ferramentas de corte, dispositivos eletrônicos e óticos e recentemente surge uma nova aplicação em eletrodos de diamante $\mathrm{CVD}^{1-3}$.

O objetivo deste trabalho é o desenvolvimento de eletrodos de diamante para utilização em eletroanálise e eletrosíntese (aparelhos opticoeletroquímicos, células fotoeletroquímicas, sensores e detetores eletroquímicos) devido ao seu alto grau de estabilidade e uma maior faixa de potencial de trabalho ${ }^{4,5}$. Para isto será necessário produzir filmes de diamante semicondutor altamente dopados com boro para diminuição de sua resistividade, pois, o diamante é um isolante ${ }^{6}$.

Os métodos normalmente empregados na dopagem de diamante CVD com boro podem ser realizados após o crescimento do filme de diamante, como a difusão térmica $^{7-9}$ e a implantação de íons ${ }^{10,11}$ ou durante o processo de crescimento que consiste na dissolução de um composto de boro num solvente. Este tipo de dopagem leva a resultados melhores em termos de homogeneidade de dopagem ao longo do volume de diamante crescido ${ }^{12,13}$. 
Alguns relatos foram feitos sobre a caracterização dos filmes de diamante através de técnicas eletroquímicas. Swain ${ }^{14}$ fêz uma comparação da resistência à corrosão entre eletrodos de diamante, grafite pirolítico altamente orientado e carbono vítreo em meio de fluoretos ácidos. Miller e colaboradores ${ }^{15}$ estudaram o comportamento dos eletrodos de diamante dopados com boro obtidos pelo método CVD em vários sistemas eletroquímicos.

Declements ${ }^{16}$ pesquisou o crescimento de filmes de diamante autosustentados em substratos de carbono vítreo (GC-20 e GC-30) através do método de deposição química a partir da fase vapor assistido por microondas, utilizando nitreto de boro (h-BNN) como fonte de difusão de boro. A evolução de $\mathrm{H}_{2}$ e $\mathrm{O}_{2}$ em eletrodos de diamante dopados com boro foi estudado por Martin ${ }^{17}$ através do método de deposição química a partir da fase vapor assistido por filamento quente.

Neste trabalho, filmes de diamante policristalino com diferentes concentrações de B/C (0, 2000 e 20000 ppm) foram preparados sobre substratos de silício através do método HFCVD. Microscopia eletrônica de varredura foi empregada para estudar a morfologia destes filmes e espectroscopia Raman foi utilizada para investigar a qualidade e nível de dopagem destes filmes.

\section{Detalhes Experimentais}

Filmes de diamante foram crescidos através de HFCVD a $750{ }^{\circ} \mathrm{C}$ de uma mistura $\mathrm{CH}_{4} / \mathrm{H}_{2}$ a uma pressão de 50 Torr. Óxido de boro $\left(\mathrm{B}_{2} \mathrm{O}_{3}\right)$ dissolvido em metanol foi utilizado como fonte de dopagem de boro. O substrato era uma pastilha de silício polida tipo-p. O sistema utilizado nos experimentos está representado na Fig. 1.

Primeiramente faz-se passar hidrogênio (1) através do borbulhador contendo óxido de boro dissolvido em metanol, o qual é arrastado numa concentração que depende da temperatura, pressão de hidrogênio no borbulhador e fluxo de hidrogênio para o interior do reator. A concentração do vapor em relação ao hidrogênio que sai do borbulhador era aproximadamente da ordem de $10 \%$, então há uma diluição em uma mistura de 0,5 vol.\% $\mathrm{CH}_{4}$ em hidrogênio (2) para crescimento de diamante de boa qualidade. O espectrômetro de massa (MS) será acoplado ao reator para análise da fase gasosa.

Foi realizado um pré-tratamento sobre a superfície do silício $\left(\sim 0,72 \mathrm{~cm}^{2}\right)$, que consiste no seu polimento com pasta de diamante $(3 \mu \mathrm{m})$ e depois lavagem com acetona em banho de ultra-som por alguns minutos e sêco ao ar. As medidas microRaman foram realizadas em um espectrômetro da Renishaw-Ramascope 2000 a temperatura ambiente utilizando a linha verde do argônio em 514,5 nm. O diâmetro nominal do feixe é da ordem de $5 \mu$ m e a resolução de $3 \mathrm{~cm}^{-1}$. Este espectrometro Raman possui uma câmara CCD que faz a amostragem do espectro completo na faixa

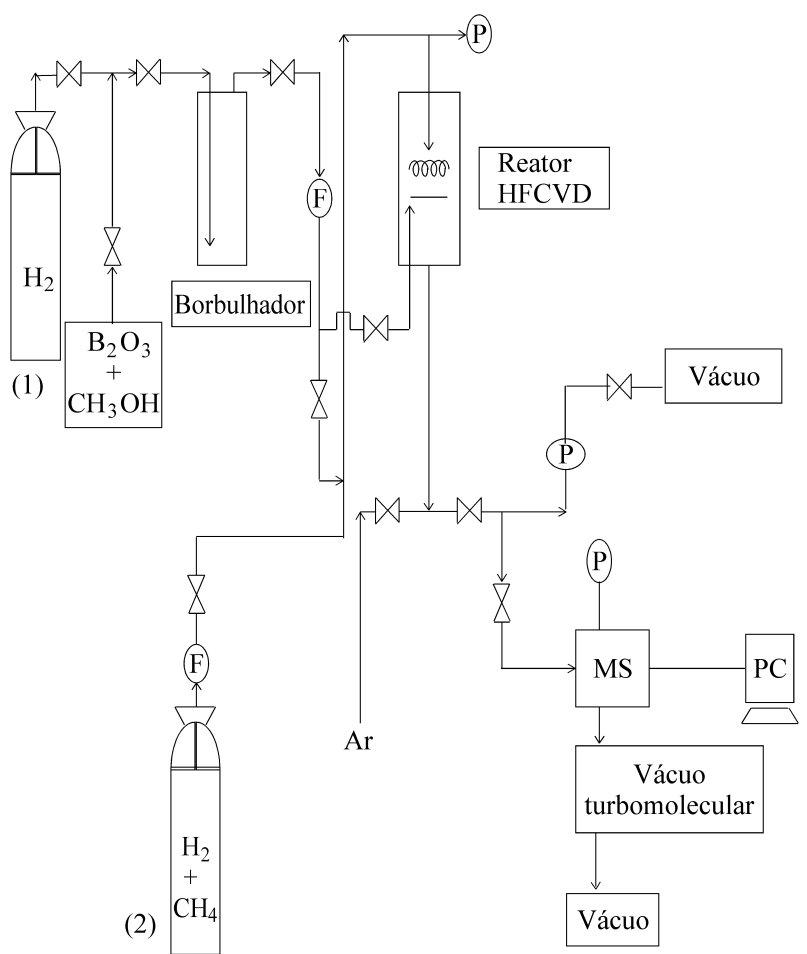

Figura 1. Sistema para crescimento de diamante com óxido de boro dissolvido em metanol.

mostrada em uma única exposição. Os espectros foram tirados em $120 \mathrm{~s}$ de amostragem.

\section{Resultados e Discussão}

\subsection{Espectroscopia Raman}

A espectroscopia Raman é geralmente utilizada na caracterização da estrutura e qualidade dos filmes de diamante sintetizados.

Uma mudança nos espectros Raman dos filmes de diamante com diferentes níveis de dopagem foi observada. As Figs. 2 a 4 apresentam os espectros dos filmes cujas concentrações foram de 0, 2000 e 20000 ppm B/C, respectivamente.

A linha característica do diamante em $1333 \mathrm{~cm}^{-1}$ se desloca para o sentido de menor energia e diminui de intensidade com o aumento do nível de dopagem do filme. Este pico quase desaparece no espectro da Fig. 4 representando o filme de maior dopagem. Por outro lado, observa-se nas Figs. 3 e 4 o aparecimento de uma banda larga em $1220 \mathrm{~cm}^{-1}$ que aumenta em intensidade com o aumento do nível de dopagem dos filmes e foi atribuído à relaxação da regra de seleção $K=0$ do espalhamento Raman devido à presença de uma concentração muito alta de $\mathrm{B}$ na rede do diamante ${ }^{18,19}$. No caso da Fig. 4 o espectro é mostrado na região extendida entre 200 e $2000 \mathrm{~cm}^{-1}$ para mostrar com maior evidência as bandas Raman geradas pela incorporação do boro. Neste caso existe uma completa relaxação da regra de seleção $\mathrm{K}=0$ e a banda larga em torno de 
$1200 \mathrm{~cm}^{-1}$ representa uma combinação de picos devidos a fonons ópticos individuais. Conforme comentado por Zhang e colaboradores ${ }^{18}$, isto é perfeitamente suportado pelos espectros Raman de $2^{\underline{a}}$ ordem do diamante.

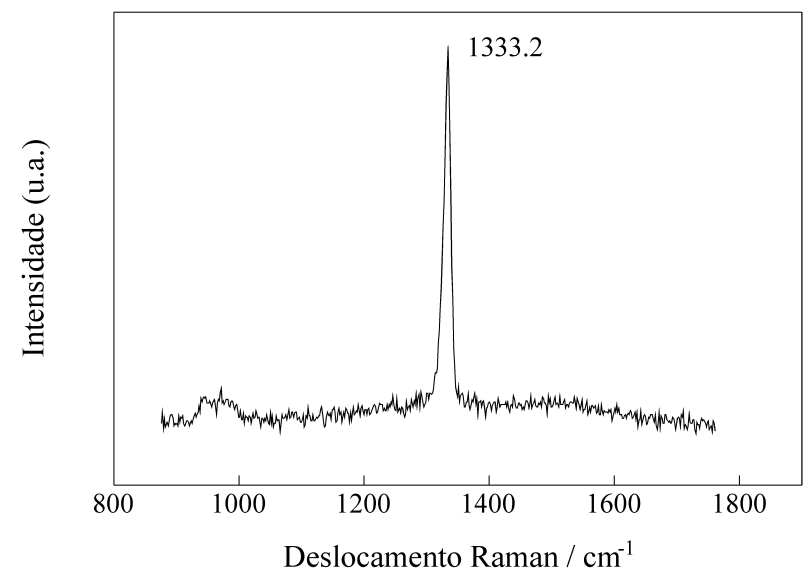

Figura 2. Espectro Raman de um filme obtido nas seguintes condições: $\mathrm{t}=3 \mathrm{~h}, \mathrm{~T}=818^{\circ} \mathrm{C}, \mathrm{B} / \mathrm{C}=0 \mathrm{ppm}$.

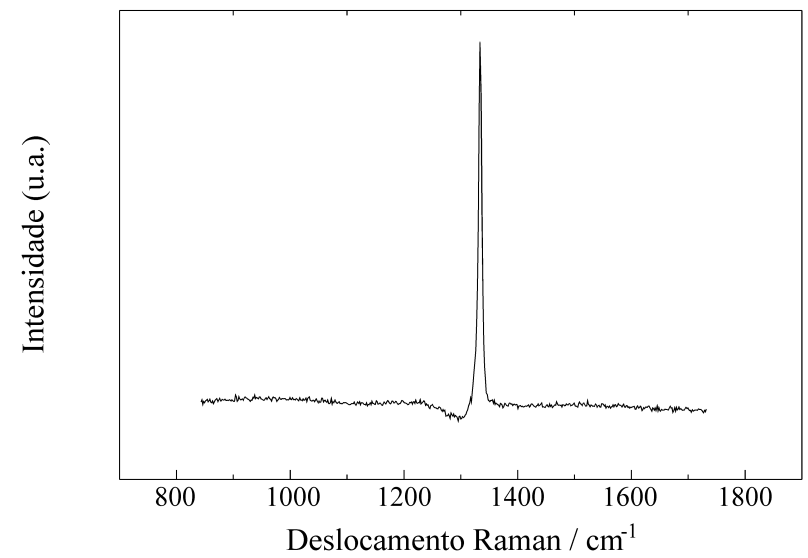

Figura 3. Espectro Raman de um filme obtido nas seguintes condições: $\mathrm{t}=4,5 \mathrm{~h}, \mathrm{~T}=818^{\circ} \mathrm{C}, \mathrm{B} / \mathrm{C}=2000 \mathrm{ppm}$.

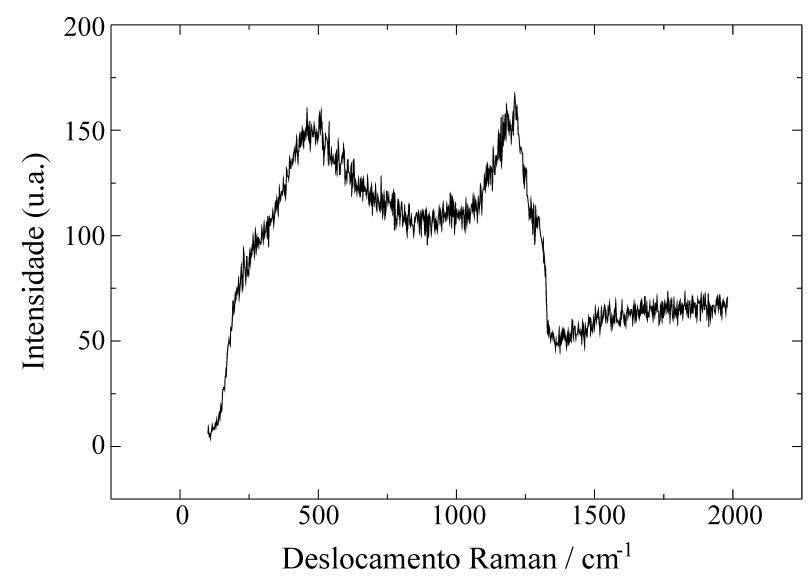

Figura 4. Espectro Raman de um filme obtido nas seguintes condições: $\mathrm{t}=4,5 \mathrm{~h}, \mathrm{~T}=670^{\circ} \mathrm{C}, \mathrm{B} / \mathrm{C}=20000 \mathrm{ppm}$.
O espectro Raman de segunda ordem é em geral similar à densidade de estados vibracionais, com a frequência dobrada, e no caso do diamante tem o pico mais intenso em $2458 \mathrm{~cm}^{-1}$, que é aproximadamente o dobro da posição do máximo da banda em $1200 \mathrm{~cm}^{-1}$.

Além disso, as bandas em 460 e $1200 \mathrm{~cm}^{-1}$ são escalonáveis a partir de espectros Raman de silício amorfo, o que pode caracterizar o espectro da Fig. 4 como sendo o espectro característico do "diamante amorfo"18.

Observando-se o espectro Raman da Fig. 4, verifica-se a partir da literatura que o nível de dopagem obtido no filme deve ser da ordem de $10^{21} \mathrm{~B} / \mathrm{cm}^{3}{ }^{18}$, equivalendo a uma resistividade da ordem de $0,1 \Omega \mathrm{cm}$. Medidas de resistência feitas diretamente com um multímetro sobre a superfície do filme indicam uma resistividade desta ordem.

\subsection{Microscopia eletrônica de varredura}

Nas Figs. 5 a 7 são apresentadas micrografias dos filmes de diamante dopados com B em diferentes concentrações (0, 2000 e $20000 \mathrm{ppm} \mathrm{B/C})$.

Observando estas micrografias, verifica-se que houve pouca variação na morfologia da superfície dos filmes. Todos os filmes apresentam uma textura uniforme contendo grãos pequenos de tamanho médio da ordem de $2 \mu \mathrm{m}$ e bem facetados.

\section{Conclusões}

Quando se aumenta o nível de dopagem de B nos filmes de diamante ocorre um deslocamento para o sentido de menor energia e uma diminuição de intensidade da linha característica do diamante em $1333 \mathrm{~cm}^{-1}$ e surgimento de uma banda extensa em $1220 \mathrm{~cm}^{-1}$.

Não foi observado variação na morfologia superficial dos filmes com diferentes níveis de dopagem de boro , 0 , 2000 e $20000 \mathrm{ppm}$ de $\mathrm{B} / \mathrm{C}$, respectivamente. O tamanho médio de grão é da ordem $2 \mu \mathrm{m}$ e bem facetado.

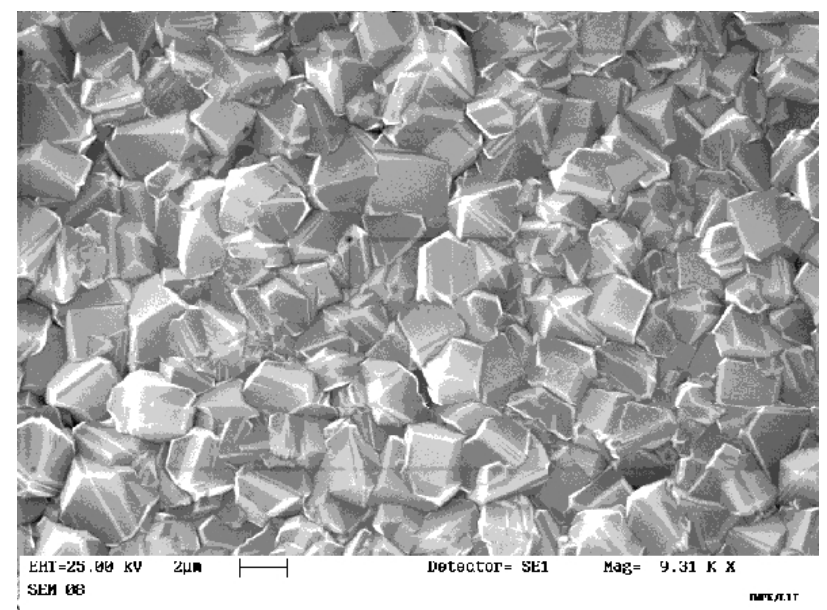

Figura 5. Microscopia Eletrônica de Varredura de um filme obtido nas seguintes condições: $\mathrm{t}=3 \mathrm{~h}, \mathrm{~T}=818^{\circ} \mathrm{C}, \mathrm{B} / \mathrm{C}=0 \mathrm{ppm}$. 


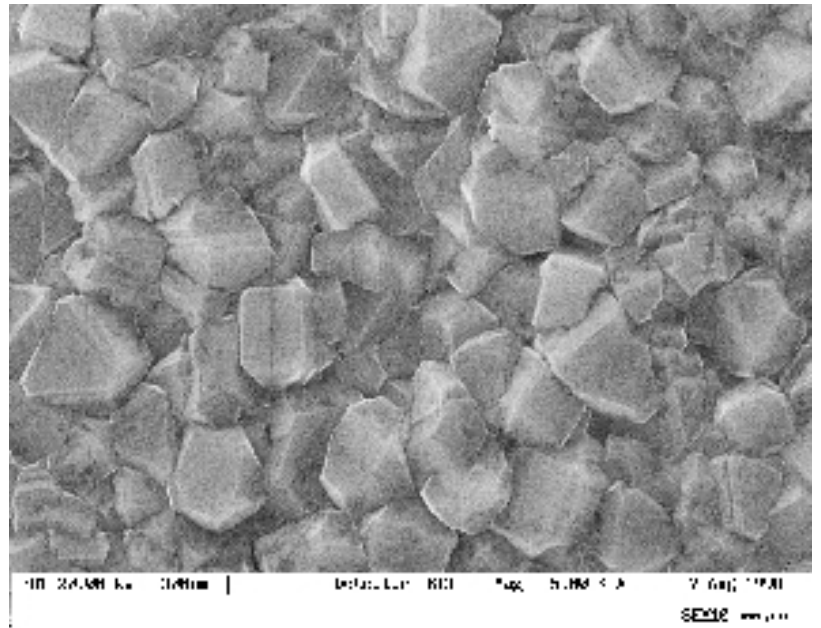

Figura 6. Microscopia Eletrônica de Varredura de um filme obtido nas seguintes condições: $\mathrm{t}=4,5 \mathrm{~h}, \mathrm{~T}=818^{\circ} \mathrm{C}, \mathrm{B} / \mathrm{C}=2000 \mathrm{ppm}$.

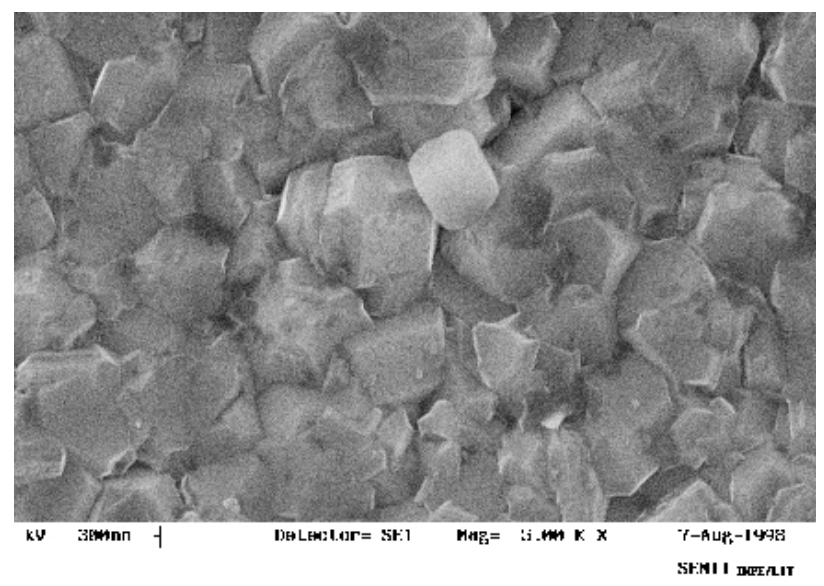

Figura 7. Microscopia Eletrônica de Varredura de um filme obtido nas seguintes condições: $\mathrm{t}=4,5 \mathrm{~h}, \mathrm{~T}=670^{\circ} \mathrm{C}, \mathrm{B} / \mathrm{C}=20000 \mathrm{ppm}$.

O filme mais dopado tem, provavelmente, um nível de dopagem da ordem de $10^{21} \mathrm{~B} / \mathrm{cm}^{3}$, ideal para a confecção dos eletrodos, que será a próxima etapa do trabalho.

\section{Bibliografia}

1. Ramesham, R.; Rose, M.F. Electrochemical characterization of doped and undoped CVD diamond deposited by microwave plasma. Diamond and Related Materials, v. 6, p. 17-27, 1997.

2. Declements, R.; Swain, G.M. The Formation and Electrochemical Activity of Microporous Diamond Thin Film Electrodes in Concentrated KOH. Journal Electrochemical Society, v. 144, p. 857-867, 1997.

3. Ramesham, R.; Rose, M.F. Cyclic voltammetric, a.c. and d.c. polarization behavior of boron-doped CVD diamond. Thin Solid Films, v. 300, p. 144-153, 1997.

4. Swain, G.M. The Use of CVD Diamond Thin Films in Electrochemical Systems. Advanced Materials, v. 6, p. 388-392, 1994.
5. Miller, B.; Kalish, R. Electrochemical Behavior of Boron-Doped Diamond Electrodes. Journal Electrochemical Society, v. 143, p. L 238-L 240, 1996.

6. Davidson, J.L. Diamond Electrical Properties and Electronic Device Behavior in Synthetic Diamond: Emerging CVD Science and Technology, Spear, K.E. Dismuskes, J.P., eds., John Wiley \& Sons, p. 355

7. Martin, H.B.; Argoitia, A.; Landau, U.; Anderson, A.B.; Angus, J.C. Hydrogen and Oxygen Evolution on Boron-Doped Diamond Electrodes. Journal Electrochemical Society, v. 143, n. 6, p. L 133-135, 1996.

8. Declements, R.; Hirsche, B.L.; Granger, M.C.; Xu, J.; Swain, G.M. Freestanding Diamond Thin Films Grown on Glassy Carbon Substrates. Journal Electrochemical Society, v. 143, n. 7, p. L 150-153, 1996.

9. Tsai, W.; Delfino, M.; Ching, L.Y.; Reynolds, G.; Hodul, D.; Cooper, C.B. Boron Doping of Diamond Via Solid State Diffusion. New Diamond Science and Technology, MRS Int. Conf. Proc. p. 937, 1991.

10. Fontaine, F.; Deneuville, A.; Gheeraert, E.; Gonon, P.; Abello, L.; Lucazeau, G. Reality of doping by boron implantation of CVD polycrystalline diamond from a comparison of Raman and electrical measurements. Diamond and Related Materials, v. 3, p. 623-627, 1994.

11. Gheeraert, E.; Fontaine, F.; Deneuville, A.; Khong, Y.L.; Collins, A.T. Characterization of defects in boron implanted chemically vapour deposited diamond films by electron paramagnetic resonance and cathodoluminescence. Diamond and Related Materials, v. 3, p. 737-740, 1994.

12. Spicka, H.; Griesser, M.; Hutter, H.; Grasserbauer, M.; Bohr, S.; Haubner, R.; Lux, B. Investigations of the incorporation of $\mathrm{B}, \mathrm{P}$ and $\mathrm{N}$ into CVD-diamond films by secondary ion mass spectrometry. Diamond and Related Materials, v. 5, p. 383-387, 1996.

13. Freitas Jr., J.A.; Doverspike, K.; Kein, P.B.; Kong, Y.L.; Collins, A.T. Luminescence studies of nitrogen and boron-doped diamond films. Diamond and Related Materials, v. 3, p. 821-824, 1994.

14. Swain, G.M. The Susceptibility to Surface Corrosion in Acidic Fluoride Media: A Comparison of Diamond, HOPG, and Glassy Carbon Electrodes. Journal Electrochemical Society, v. 141, n. 12, p. 3382-3393, 1996.

15. Vinokur, N.; Miller, B.; Avyigal Y.; Kalish, R. Electrochemical Behavior of Boron-Doped Diamond Electrodes. Journal Electrochemical Society, v. 143, n. 10, p. L 238-240, 1996. 
16. Declements, R.; Hirsche, B.L.; Granger, M.C.; Xu, J.; Swain, G.M. Freestanding Diamond Thin Films Grown on Glassy Carbon Substrates. Journal Electrochemical Society, v. 143, n. 7, p. L 150-153, 1996.

17. Martin, H.B.; Argoitia, A.; Landau, U.; Anderson, A.B.; Angus, J.C. Hydrogen and Oxygen Evolution on BoronDoped Diamond Electrodes. Journal Electrochemical Society, v. 143, n. 6, p. L 133-135, 1996.
18. Zhang, R.J.; Lee, S.T.; Lam, Y.W. Characterization of heavily boron-doped diamond films. Diamond Related Materials, v. 5, p. 1288-1294, 1996.

19. Colineau, E.; Gheeraert, E.; Deneuville, A. Minimization of the defects concentration from boron incorporation in polycrystalline diamond films. Diamond Related Materials, v. 6, p. 778-782, 1997. 